\title{
FOURIER INTEGRALS AND METRIC GEOMETRY
}

\author{
BY
}

\author{
J. VON NEUMANN AND I. J. SCHOENBERG
}

\section{INTRODUCTION}

1. Let $S$ be a metric space, the distance $d(f, g)$ between two elements of $S$ satisfying the usual postulates. Let $f_{t}(-\infty<t<\infty)$ be a function whose values lie in $S$ and which is metrically continuous, that is, $d\left(f_{t+h}, f_{t}\right) \rightarrow 0$ if $h \rightarrow 0$. If this function has the property that

$$
d\left(f_{t}, f_{s}\right)=F(t-s)
$$

is a function of the difference $t-s$ only, we call the curve $\Gamma$ in $S$ defined by $f_{t}$ $(-\infty<t<\infty)$ a screw line of $S$ and $F(t)=d\left(f_{t}, f_{0}\right)$ a screw function of $S$. The reason for this terminology is as follows. If $\tau$ is a real parameter, the two curves $\Gamma_{0}: f_{t}(-\infty<t<\infty)$ and $\Gamma_{\tau}: f_{t+\tau}(-\infty<t<\infty)$ which are identical as point sets, are isometrically mapped on each other by the correspondence $f_{t} \leftrightarrow f_{t+\tau}$, for

$$
d\left(f_{t}, f_{s}\right)=F(t-s)=d\left(f_{t+\tau}, f_{s+\tau}\right),
$$

in view of (0.1). These congruent mappings of $\Gamma$ into itself form a one-parameter group.

The following properties of a screw function $F(t)$ of $S$ are obvious: $F(t)$ is a continuous non-negative even function and $F(0)=0$; if $F(t)$ is a screw function, then all functions $F(k t)$ ( $k$ real) are screw functions.

A different point of view which puts the emphasis on the screw function $F(t)$ rather than on the screw line $\Gamma$ is as follows. Consider the real axis $-\infty<t<\infty$ as a euclidean space $E_{1}$ and change its metric from $|t-s|$ to $F(t-s)$. We thus get a new space which, following Blumenthal, we shall call the metric transform of $E_{1}$ by $F(t)$ and denote by $F\left(E_{1}\right)$. For what functions $F(t)$ may this metric transform $F\left(E_{1}\right)$ be isometrically imbedded in $S$ ? Clearly $F\left(E_{1}\right)$ enjoys this property if and only if $F(t)$ is a screw function of $S$. For if the mapping of the point $t$, of $F\left(E_{1}\right)$, into the point $f_{t}$, of $S$, performs the imbedding of $F\left(E_{1}\right)$ into $S$, then (0.1) expresses the isometricity of this imbedding.

Presented to the Society, September 1, 1936, and December 29, 1938; received by the editors September 23, 1940. The first communication to the Society [Bulletin of the American Mathematical Society, abstract 42-9-353] covered the contents of Part I and Part II of the present paper; the contents of Part III were the subject of the second communication of 1938 [abstract 47-1-47]. Thus the contents of Parts I and II precede in time the articles [4] and [5], listed in the bibliography at the end of this paper, which were partly suggested by this earlier work frequently referred to in these articles. Part III, however, carries on the work presented in [4] and [5] and is essentially based on some of that work. 
2. As examples we mention

$$
F_{3}(t)=\left(t^{2}+\sin ^{2} t\right)^{1 / 2}
$$

which is a screw function of the euclidean space $E_{3}$, in view of the identity $F_{3}^{2}(t-s)=(t-s)^{2}+\sin ^{2}(t-s)$

$$
=(t-s)^{2}+\frac{1}{4}(\cos 2 t-\cos 2 s)^{2}+\frac{1}{4}(\sin 2 t-\sin 2 s)^{2} .
$$

Similarly

$$
F_{2 N}(t)=\left(\sum_{\nu=1}^{N} A_{\nu} \sin ^{2} u_{\nu} t\right)^{1 / 2}, \quad A_{\nu}>0,0<u_{1}<u_{2}<\cdots<u_{N}
$$

is a screw function of $E_{2 N}$, as seen from

$$
F_{2 N}^{2}(t-s)=\sum_{\nu=1}^{N}\left\{\frac{1}{4} A_{\nu}\left(\cos 2 u_{\nu} t-\cos 2 u_{\nu} s\right)^{2}\right.
$$

Finally

$$
\left.+\frac{1}{4} A_{\nu}\left(\sin 2 u_{\nu} t-\sin 2 u_{\nu} s\right)^{2}\right\} .
$$

$$
\begin{aligned}
& F_{2 N-1}(t)=\left(C t^{2}+\sum_{\nu=1}^{N-1} A_{\nu} \sin ^{2} u_{\nu} t\right)^{1 / 2}, \\
& \quad C>0, A_{\nu}>0,0<u_{1}<\cdots<u_{N-1},
\end{aligned}
$$

is a screw function of $E_{2 N-1}$, as shown by

$$
\begin{aligned}
F_{2 N-1}^{2}(t-s) & =C(t-s)^{2} \\
+ & \sum_{\nu=1}^{N-1}\left\{\frac{1}{4} A_{\nu}\left(\cos 2 u_{\nu} t-\cos 2 u_{\nu} s\right)^{2}+\frac{1}{4} A_{\nu}\left(\sin 2 u_{\nu} t-\sin 2 u_{\nu} s\right)^{2}\right\} .
\end{aligned}
$$

We shall see that (0.2) and (0.3) are the most general screw functions of $E_{2 N}$ and $E_{2 N-1}$, respectively, which are not also screw functions of a euclidean space of lower dimensions.

The starting point of the present investigation was W. A. Wilson's recent remark that if

$$
F(t)=|t|^{1 / 2}
$$

then the metric transform $F\left(E_{1}\right)$ is imbeddable in the Hilbert space $\mathfrak{S}$, hence

$$
F^{2}(t-s)=|t-s|=\left\|f_{t}-f_{s}\right\|^{2},
$$

for a suitable function $f_{t}(-\infty<t<\infty)$ with values in $\mathfrak{S}[8$, p. 64]. According to our present point of view $(0.4)$ is a screw function of $\mathfrak{S}$. We shall see that this $F(t)$ is not a screw function of any euclidean space.

3. The principal purpose of this paper is to determine all screw functions of Hilbert space. It consists of three parts. In Part I we state our fundamental 
result (Theorem 1) and show by means of elementary results of Menger and Schoenberg that all functions $F(t)$ there described are screw functions of $\mathfrak{S}$. Furthermore, those screw functions of $\mathfrak{S}$ are characterized which correspond to screw lines $\Gamma$ with one of the following properties: $\Gamma$ is euclidean, bounded, rectifiable or closed.

The converse statement to the effect that Theorem 1 yields all screw functions of $\mathfrak{S}$ is established in two essentially different ways in Part II and Part III respectively. In Part II this is proved by a direct investigation of the group of isometric mappings of $\mathfrak{S}$ into itself which is induced by the group of isometric mappings of a screw line into itself. Free use is made of the theory of Hermitian operators in Hilbert space. In Part III $\mathfrak{S}$ intervenes only by its metric in accordance with the ideas of Menger on the metric characterization of metric spaces. The method used is an elaboration of the metrical approach of Part I which is made more effective by an appeal to the theory of positive definite functions, i.e., the characteristic functions of the theory of probabilities. This connection was pointed out in two recent papers by one of us $[4,5]$. In $[5$, p. 837], it was shown that the question as to when the metric transform $F\left(E_{m}\right)$ is imbeddable in $\mathfrak{S}$ depended on certain limit (closure) theorems. Here we establish these theorems in a form similar to P. Lévy's limit theorem concerning characteristic functions.

In conclusion we want to say that the operator method of Part II, dealing with the imbedding of $F\left(E_{1}\right)$ in $\mathfrak{H}$, may also be extended to cover the general case of the imbedding of $F\left(E_{m}\right)$ in $\mathfrak{S}$. Although this extension has been fully worked out, for reasons of conciseness we treat in Part II only the case of screw lines $(m=1)$.

\section{Part I. The FUndamental Theorem ON SCREW FUnCtions IN HilbERT SPACE AND ELEMENTARY CONSEQUENCES}

1.1. Let $\mathfrak{S}$ be a real Hilbert space. For every $t>-\infty$, and $\langle+\infty$, let a point $f_{t}$ of $\mathfrak{S}$ be given, such that

(i) $f_{t}$ is a metrically continuous function of $t$,

(ii) the distance of $f_{t}$ and $f_{s}$ depends on $t-s$ only.

According to the general definition of our Introduction, the curve $\Gamma: f_{t}$ is a screw line of $\mathfrak{S}$. Condition (ii) means

$$
\left\|f_{t}-f_{s}\right\|=F(t-s) \text {. }
$$

Then (i) merely states that

$$
F(t) \rightarrow 0 \text { if } t \rightarrow 0,
$$

and conversely, (1.2) implies the continuity of $f_{t}$ and therefore of $F(t)$ everywhere. $F(t)$ is called a screw function of $\mathfrak{S}$.

Let $f_{t_{0}}, f_{t_{1}}, \cdots, f_{t_{n}}\left(t_{0}=0\right)$ be $n+1$ points of $\Gamma$. By (1.1) their mutual distances are $\left\|f_{t_{i}}-f_{t_{k}}\right\|=F\left(t_{i}-t_{k}\right)$. As these points may be thought of as lying 
in a $n$-dimensional linear subspace of $\mathfrak{S}$, i.e., a $E_{n}$, we have by a known theorem [3, Theorem 1]

$$
\sum_{i, k=1}^{n}\left\{F^{2}\left(t_{i}\right)+F^{2}\left(t_{k}\right)-F^{2}\left(t_{i}-t_{k}\right)\right\} \rho_{i} \rho_{k} \geqq 0,
$$

for arbitrary real $\rho_{i}$. Conversely, let $F(t)$ be a continuous even non-negative function, with $F(0)=0$, enjoying the property (1.3) for arbitrary real $t_{i}, \rho_{i}$ $(n=2,3, \cdots)$. Geometrically this means: The points $t_{0}=0, t_{1}, \cdots, t_{n}$, of the space $F\left(E_{1}\right)$, which is obtained from $E_{1}$ by changing its metric from $|t-s|$ to $F(t-s)$, may be imbedded in $E_{n}$. By a theorem of Menger [2] this is sufficient to insure the possibility of imbedding $F\left(E_{1}\right)$ in $\mathfrak{S}$. We can therefore state

LemMa 1. A continuous even non-negative function $F(t)$ vanishing at the origin is a screw function of $\mathfrak{S}$ if and only if it satisfies the inequality (1.3) for arbitrary real $t_{i}, \rho_{i}$ and for $n=2,3,4, \cdots$.

The interest of this analytical characterization of screw function lies in its obvious consequence that the squares $F^{2}(t)$ of screw functions form a convex class of functions: If $F_{1}^{2}(t)$ and $F_{2}^{2}(t)$ are squares of screw functions then also $F_{1}^{2}(t)+F_{2}^{2}(t)$ is the square of a screw function. In view of the euclidean screw functions $(0.2),(0.3)$, exhibited in the Introduction, this convexity property suggests the following explicit expression of the screw functions of $\mathfrak{S}$.

TheOREM 1. (Fundamental theorem.) The class of screw functions $F(t)$ of Hilbert space is identical with the class of functions whose squares are of the form

$$
F^{2}(t)=\int_{0}^{\infty} \frac{\sin ^{2} t u}{u^{2}} d \gamma(u),
$$

where $\gamma(u)$ is non-decreasing for $u \geqq 0$ and such that

$$
\int_{1}^{\infty} u^{-2} d \gamma(u) \text { exists. }
$$

A proof that all functions $F(t)$ furnished by (1.4) are screw functions is exceedingly simple. For it suffices to show that $F^{2}(t)$ satisfies the inequality (1.3) (Lemma 1). Indeed, in view of the identity

$$
\sin ^{2} t_{i} u+\sin ^{2} t_{k} u-\sin ^{2}\left(t_{i}-t_{k}\right) u=2 \sin ^{2} t_{i} u \sin ^{2} t_{k} u+\frac{1}{2} \sin 2 t_{i} u \sin 2 t_{k} u,
$$

we have

$$
\begin{aligned}
& \sum_{i, k=1}^{n}\left\{F^{2}\left(t_{i}\right)+F^{2}\left(t_{k}\right)-F^{2}\left(t_{i}-t_{k}\right)\right\} \rho_{i} \rho_{k} \\
& \quad=\int_{0}^{\infty}\left\{2\left(\sum_{1}^{n} \rho_{i} \sin ^{2} t_{i} u\right)^{2}+\frac{1}{2}\left(\sum_{1}^{n} \rho_{i} \sin 2 t_{i} u\right)^{2}\right\} u^{-2} d \gamma(u) \geqq 0 .
\end{aligned}
$$


The converse statement that the square of a screw function is necessarily of the form (1.4) is proved in two different ways in Part II and Part III of this paper.

1.2. Let us now see which of the screw functions of $\mathfrak{S}$ are euclidean screw functions, that is, belong to some euclidean space. It is convenient for this purpose to write (1.4) in the form

$$
F^{2}(t)=C t^{2}+\int_{+0}^{\infty} \frac{\sin ^{2} t u}{u^{2}} d \gamma(u), \quad C=\gamma(+0)-\gamma(0) .
$$

TheOREM 2. A screw function $F(t)$ defined by (1.4) is euclidean if and only if $\gamma(u)$ has a finite number $N$ of points of increase. $F(t)$ belongs to $E_{m}$ and to no lower space $E_{m^{\prime}}\left(m^{\prime}<m\right)$ if and only if

$$
\begin{array}{lll}
C=0, & N=m / 2 \quad \text { for } m \text { even, } \\
C>0, & N=(m+1) / 2 \text { for } m \text { odd. }
\end{array}
$$

Indeed, let $N$ be finite. If $C=0$, then $F(t)$ is of the form (0.2), and $u_{1}, \cdots, u_{N}$ are precisely the points of increase of $\gamma(u)$. We know by $\left(0.2^{\prime}\right)$. that $F(t)$ is a screw function of $E_{2 N}$. Moreover its screw line

$$
\left(\frac{1}{2} A_{\nu}^{1 / 2} \cos 2 u_{\nu} t, \frac{1}{2} A_{\nu}^{1 / 2} \sin 2 u_{\nu} t\right), \quad \nu=1,2, \cdots, N ;-\infty<t<\infty,
$$

lies in $E_{2 N}$ but in no linear subspace of $E_{2 N}$. Similarly, if $C>0$, it is shown by (0.3), $\left(0.3^{\prime}\right)$, that $F(t)$ belongs to $E_{m}, m=2 N-1$.

Let now $\gamma(u)$ have infinitely many points of increase. We want to show that $F(t)$ is not euclidean, that is, the metric transform $F\left(E_{1}\right)$ cannot be imbedded in a euclidean space. For if $n$ is an arbitrary positive integer, we may find $n$ points of increase $u=u_{\nu}(\nu=1, \cdots, n)$ of $\gamma(u)$, such that $0<u_{1}<u_{2}<\cdots<u_{n}$. We shall now locate in $F\left(E_{1}\right) n+1$ points $t=0$, $t_{1}, \cdots, t_{n}$, which cannot be imbedded in $E_{n-1}$. Such points will enjoy this property if the quadratic form (1.6) is positive definite [3, Theorem 1$]$. The linear independence of the functions $\sin 2 t u_{1}, \cdots, \sin 2 t u_{n}$, implies the existence of values $t_{i}$, such that

$$
\operatorname{det}\left\|\sin \left(2 t_{i} u_{k}\right)\right\|_{1, n} \neq 0 .
$$

The form (1.6) is now positive definite; for otherwise there would exist values $\rho_{i}$, with

$$
\sum \underset{\rho_{i}}{2}>0
$$

which make the form (1.6) vanish. But the vanishing of the integral of (1.6) implies the vanishing of its integrand at all points of increase of $\gamma(u)$, hence in particular 


$$
\sum_{i=1}^{n} \rho_{i} \sin \left(2 t_{i} u_{k}\right)=0
$$$$
k=1, \cdots, n \text {. }
$$

The incompatibility of the three relations (1.10), (1.11), (1.12) completes this indirect proof ; for as $n$ may be taken arbitrarily large, $F(t)$ is not euclidean.

Wilson's screw function (0.4) is of the form (1.4) since

$$
F^{2}(t)=|t|=\frac{2}{\pi} \int_{0}^{\infty} \frac{\sin ^{2} t u}{u^{2}} d u .
$$

Since $\gamma(u)=2 u / \pi$ has infinitely many points of increase, it is not a euclidean screw function. A somewhat more general example is

$$
F(t)=|t| \kappa,
$$

$$
0<\kappa<1
$$

Indeed

$$
F^{2}(t)=|t|^{2 \kappa}=\int_{0}^{\infty} \sin ^{2} t u \cdot u^{-1-2 \kappa} d u / \int_{0}^{\infty} \sin ^{2} u \cdot u^{-1-2 \kappa} d u, \quad 0<\kappa<1,
$$

is of the form (1.4).

1.3. Let us find conditions which insure the boundedness of a screw function $F(t)$. Later we shall see that this occurs when the corresponding screw line lies on a sphere of $\mathfrak{S}$. For the present we prove

THEOREM 3. The screw function $F(t)$ given by

$$
F^{2}(t)=C t^{2}+\int_{+0}^{\infty} \frac{\sin ^{2} t u}{u^{2}} d \gamma(u), \quad C \geqq 0,
$$

is bounded if and only if

$$
C=0 \text { and } \int_{+0}^{\infty} u^{-2} d \gamma(u) \text { exists. }
$$

More precisely, if $C=0$, we have

$$
\frac{1}{2} \int_{+0}^{\infty} \frac{d \gamma(u)}{u^{2}} \leqq \limsup _{t \rightarrow \infty} F^{2}(t) \leqq \int_{+0}^{\infty} \frac{d \gamma(u)}{u^{2}},
$$

where all three members may also be infinite.

As $C=0$ is obviously necessary in order that $F^{2}(t)$ be bounded, it suffices to prove the inequalities (1.17). Let

$$
F_{\epsilon, a}^{2}(t)=\int_{\epsilon}^{a} \frac{\sin ^{2} t u}{u^{2}} d \gamma(u), \quad 0<\epsilon<a<+\infty .
$$


We have

$$
\begin{aligned}
\frac{1}{T} \int_{0}^{T} F_{\epsilon, a}^{2}(t) d t & =\int_{\epsilon}^{a}\left(\frac{1}{T} \int_{0}^{T} \sin ^{2} t u d t\right) u^{-2} d \gamma(u) \\
& =\int_{\epsilon}^{a}\left(\frac{1}{2}-\frac{\sin 2 T u}{4 T u}\right) u^{-2} d \gamma(u)
\end{aligned}
$$

hence

$$
\lim _{T \rightarrow \infty} \frac{1}{T} \int_{0}^{T} F_{\epsilon, a}^{2}(t) d t=\frac{1}{2} \int_{\epsilon}^{a} u^{-2} d \gamma(u)
$$

Whence, since $F^{2}(t) \geqq F_{\epsilon, a}^{2}(t)$, we derive

$$
\limsup _{t \rightarrow \infty} F^{2}(t) \geqq \limsup _{t \rightarrow \infty} F_{\epsilon, a}^{2}(t) \geqq \frac{1}{2} \int_{\epsilon}^{a} u^{-2} d \gamma(u) .
$$

By comparing the extreme terms only and allowing $\epsilon \rightarrow 0$ and $a \rightarrow \infty$, we get the first inequality (1.17). The second inequality (1.17) follows from

$$
F^{2}(t)=\int_{+0}^{\infty} \frac{\sin ^{2} t u}{u^{2}} d \gamma(u) \leqq \int_{+0}^{\infty} \frac{1}{u^{2}} d \gamma(u) .
$$

The euclidean screw functions (0.2) are always bounded; $(0.3)$ are never bounded.

1.4. We shall now investigate when a screw line $\Gamma$ of a screw function $F(t)$ is rectifiable. With this purpose in view we show first that

$$
\lim _{t \rightarrow 0}\left(\frac{F(t)}{t}\right)^{2}=\int_{0}^{\infty} d \gamma(u),
$$

where both sides may also be infinite.

Indeed, to each $\epsilon>0$ there corresponds a $\delta>0$, such that $\sin x^{2} / x^{2}>1-\epsilon$ if $x \geqq 0$ and $\leqq \delta$, hence $\sin ^{2} t u /(t u)^{2}>1-\epsilon$ if $0 \leqq u \leqq \delta t^{-1}, t>0$. But then

$$
\left(\frac{F(t)}{t}\right)^{2}=\int_{0}^{\infty} \frac{\sin ^{2} t u}{t^{2} u^{2}} d \gamma(u) \geqq \int_{0}^{\delta t^{-1}} \frac{\sin ^{2} t u}{t^{2} u^{2}} d \gamma(u) \geqq(1-\epsilon) \int_{0}^{\delta t^{-1}} d \gamma(u),
$$

and therefore

$$
\liminf _{t \rightarrow 0}\left(\frac{F(t)}{t}\right)^{2} \geqq(1-\epsilon) \int_{0}^{\infty} d \gamma(u) .
$$

On allowing $\epsilon \rightarrow 0$, we have

$$
\liminf _{t \rightarrow 0}\left(\frac{F(t)}{t}\right)^{2} \geqq \int_{0}^{\infty} d \gamma(u)
$$


This, and the obvious inequality

$$
\left(\frac{F(t)}{t}\right)^{2}=\int_{0}^{\infty} \frac{\sin ^{2} t u}{t^{2} u^{2}} d \gamma(u) \leqq \int_{0}^{\infty} d \gamma(u)
$$

imply (1.18).

Let now $t_{0}=0<t_{1}<\cdots<t_{n-1}<t_{n}=t$ be a subdivision of the fixed interval $(0, t)$ and $\delta=\max \left(t_{i}-t_{i-1}\right)$. (1.1) and (1.18) now imply

$$
\begin{aligned}
\lim _{\delta \rightarrow 0} \sum_{i=1}^{n}\left\|f_{t_{i}}-f_{t_{i-1}}\right\| & =\lim _{\delta \rightarrow 0} \sum_{1}^{n} F\left(t_{i}-t_{i-1}\right) \\
& =\lim _{\delta \rightarrow 0} \sum_{1}^{n} \frac{F\left(t_{i}-t_{i-1}\right)}{t_{i}-t_{i-1}} \cdot\left(t_{i}-t_{i-1}\right)=t \cdot\left(\int_{0}^{\infty} d \gamma(u)\right)^{1 / 2} .
\end{aligned}
$$

This proves the following theorem.

Theorem 4. A screw line $f_{t}(-\infty<t<\infty)$ corresponding to the screw function $F(t)$ defined by (1.4) is rectifiable if and only if $\gamma(u)$ is bounded. The length $s$ of the arc $(0, t)$ of the screw line is then connected with the parameter $t$ by the relation

$$
s=t(\gamma(\infty)-\gamma(0))^{1 / 2}=t \cdot \lim _{\tau \rightarrow 0} \frac{F(\tau)}{\tau} .
$$

In particular, if $\gamma(\infty)-\gamma(0)=1, t$ is identical with the length of arc along the screw line of $F(t)$.

The euclidean screw lines corresponding to $(0.2),(0.3)$ are always rectifiable, the relations between $s$ and $t$ being respectively

$$
s=t\left(\sum_{1}^{N} A_{\nu} u_{\nu}^{2}\right)^{1 / 2}, \quad s=t\left(C+\sum_{1}^{N-1} A_{\nu} u_{\nu}^{2}\right)^{1 / 2} .
$$

The screw lines of (1.14) are non-rectifiable, since $F(t) / t \rightarrow \infty$ as $t \rightarrow 0$.

1.5. When is a screw line $\Gamma$, corresponding to the screw function $F(t)$, a. closed curve? Let $f_{t_{0}}$ be a double point of $\Gamma$, that is, $f_{t_{0}}=f_{t_{0}+\tau}$ for some $\tau>0$. Then $0=\left\|f_{t_{0}+\tau}-f_{t_{0}}\right\|=F(\tau)$, hence $\left\|f_{t+\tau}-f_{t}\right\|=F(\tau)=0$ or $f_{t+\tau}=f_{t}$ for all real $t$. This means that $f_{t}$ and therefore also $F(t)=\left\|f_{t}-f_{0}\right\|$ has the period $\tau$ and $\Gamma$ is a closed curve. Conversely, all this is implied if $F(t)$ is periodic. Let this be the case and let $\tau$ be the least positive period of $F(t)$, which exists if $F(t) \not \equiv 0$. From (1.7) we get

$$
F^{2}(\tau)=C \tau^{2}+\int_{+0}^{\infty} \frac{\sin ^{2} \tau u}{u^{2}} d \gamma(u)=0 .
$$

This implies that $C=0$ and that $\gamma(u)$ is constant in all the intervals 
$(k-1) \pi / \tau<u<k \pi / \tau(k=1,2,3, \cdots)$ where $\sin ^{2} \tau u>0$. But then $F^{2}(t)$ reduces to the series

$$
F^{2}(t)=\sum_{k=1}^{\infty} c_{k} \sin ^{2}\left(\frac{k \pi}{\tau} t\right), \quad \tau>0, c_{k} \geqq 0, \sum_{1}^{\infty} c_{k} \text { convergent. }
$$

TheOREM 5. A screw line of $\mathfrak{S}$ is a closed curve if and only if its screw function is periodic and its square is of the form (1.20).

The screw lines of (1.20) are rectifiable if and only if $\sum_{1}^{\infty} k^{2} c_{k}$ converges.

A euclidean screw line is closed only if it is even-dimensional, that is, only if the form $(0.2)$ and its frequencies $u_{\nu}(\nu=1, \cdots, N)$ have rational $\operatorname{ratios}\left({ }^{1}\right)$.

The Fourier series developments of the Bernoulli polynomials $B_{2}(t), B_{4}(t)$ furnish the following simple examples of periodic screw functions of period one:

$$
\begin{array}{r}
F_{1}^{2}(t)=t(1-t)=\frac{1}{6}-B_{2}(t)=\sum_{1}^{\infty} \frac{1-\cos 2 \pi n t}{n^{2} \pi^{2}}=\frac{2}{\pi^{2}} \sum_{1}^{\infty} \frac{\sin ^{2} \pi n t}{n^{2}} \\
0 \leqq t \leqq 1 \\
F_{2}^{2}(t)=t^{2}(1-t)^{2}=\frac{1}{30}+B_{4}(t)=3 \sum_{1}^{\infty} \frac{1-\cos 2 \pi n t}{n^{4} \pi^{4}}=\frac{6}{\pi^{4}} \sum_{1}^{\infty} \frac{\sin ^{2} \pi n t}{n^{4}} \\
0 \leqq t \leqq 1
\end{array}
$$

The screw lines of $F_{1}(t)$ are non-rectifiable; those of $F_{2}(t)$ are rectifiable with $t$ as length of arc, since $\lim _{t \rightarrow 0} F_{2}(t) / t=1$.

1.6. We conclude this survey of these most elementary and characteristic features of screw lines by proving the following theorem.

Theorem 6. A screw line $\Gamma$ of $\mathfrak{S}$ is bounded if and only if it can be placed on a sphere of $\mathfrak{S}$.

The sufficiency of the condition is clear. Conversely, let the screw line and therefore also its screw function $F(t)$ be bounded. By Theorem 3 we have

Setting

$$
F^{2}(t)=\int_{+0}^{\infty} \frac{\sin ^{2} t u}{u^{2}} d \gamma(u) .
$$

$$
\alpha(0)=0, \quad \alpha(u)=\int_{+0}^{u} u^{-2} d \gamma(u), \quad u>0
$$

(1) The projections of such screw lines on any of the coordinate planes $x_{i} O x_{k}$ are closed Lissajou curves. Conversely, any Lissajou curve whether open or closed may be regarded as a plane projection of a screw line of $E_{4}$. 
we get

$$
F^{2}(t)=\int_{0}^{\infty} \sin ^{2} t u d \alpha(u)
$$

where $\alpha(u)$ is non-decreasing and bounded. Define $r>0$ by

$$
4 r^{2}=\alpha(\infty) .
$$

We shall prove that $\Gamma$ can be placed on a sphere of $\mathfrak{S}$ of radius $r$. Consider the metric transform $F\left(E_{1}\right)$, i.e., the real axis $-\infty<t<\infty$ with the distance $d\left(t, t^{\prime}\right)=F\left(t-t^{\prime}\right)$; add to this space a new point $A$ and complete the definition of distance throughout $F\left(E_{1}\right)+A$ by setting

$$
d(A, t)=r .
$$

Not only $F\left(E_{1}\right)$ can be inbedded in $\mathfrak{S}$, as shown by (1.6), but also $F\left(E_{1}\right)+A$ may so be imbedded. It suffices to show that the points $A, t_{1}, \cdots, t_{n}$ may be placed in $E_{n}$. Indeed, we have in view of (1.22), (1.23), (1.24)

$$
\begin{aligned}
\sum_{i, k=1}^{n}\left(d^{2}\left(A, t_{i}\right)\right. & \left.+d^{2}\left(A, t_{k}\right)-d^{2}\left(t_{i}, t_{k}\right)\right) \rho_{i} \rho_{k} \\
= & \sum_{1}^{n}\left(r^{2}+r^{2}-F^{2}\left(t_{i}-t_{k}\right)\right) \rho_{i} \rho_{k} \\
= & \sum_{1}^{n}\left\{2 r^{2}-\frac{1}{2} \int_{0}^{\infty}\left[1-\cos 2\left(t_{i}-t_{k}\right) u\right] d \alpha(u)\right\} \rho_{i} \rho_{k} \\
= & \frac{1}{2} \int_{0}^{\infty}\left\{\sum_{1}^{n} \cos 2\left(t_{i}-t_{k}\right) u \cdot \rho_{i} \rho_{k}\right\} d \alpha(u) \\
& =\frac{1}{2} \int_{0}^{\infty}\left\{\left(\sum_{1}^{n} \rho_{i} \cos 2 t_{i} u\right)^{2}+\left(\sum_{1}^{n} \rho_{i} \sin 2 t_{i} u\right)^{2}\right\} d \alpha(u) \geqq 0 .
\end{aligned}
$$

Hence $F\left(E_{1}\right)+A$ may be imbedded in $\mathfrak{S}$ by Menger's theorem. Now (1.24) shows that $F\left(E_{1}\right)$ is mapped into a screw line $\Gamma$ lying on the sphere of radius $r$ whose center is the image of $A$.

\section{Part II. First proof of the fundamental theorem BY THE THEORY OF OPERATORS}

2.1. Let $f_{t}(-\infty<t<\infty)$ be a screw line of $\mathfrak{S}$, and $F(t)$ the corresponding screw function. For greater generality we assume that $\mathfrak{S}$ is a real complete unitary space (not necessarily separable). As in $\$ 1.1$ we have

$$
\begin{gathered}
\left\|f_{t}-f_{s}\right\|=F(t-s), \\
F(t) \rightarrow 0 \text { if } t \rightarrow 0 .
\end{gathered}
$$


Using the reality of $\mathfrak{S}$ we have

$$
\begin{aligned}
\left(f_{t}-f_{u}, f_{s}-f_{u}\right) & =\frac{1}{2}\left\{\left\|f_{t}-f_{u}\right\|^{2}+\left\|f_{s}-f_{u}\right\|^{2}-\left\|\left(f_{t}-f_{u}\right)-\left(f_{s}-f_{u}\right)\right\|^{2}\right\} \\
& =\frac{1}{2}\left\{\left\|f_{t}-f_{u}\right\|^{2}+\left\|f_{s}-f_{u}\right\|^{2}-\left\|f_{t}-f_{s}\right\|^{2}\right\} \\
& =\frac{1}{2}\left\{F^{2}(t-u)+F^{2}(s-u)-F^{2}(t-s)\right\} .
\end{aligned}
$$

Hence setting

$$
F^{2}(p)+F^{2}(q)-F^{2}(p-q)=2 G(p, q)
$$

we have

$$
\left(f_{t}-f_{u}, f_{s}-f_{u}\right)=G(t-u, s-u) .
$$

Let $\mathfrak{W}_{1}$ be the closed linear subset of $\mathfrak{E}$, which is spanned by all

$$
f_{t}-f_{0},
$$

$t$ rational.

Write all rational numbers as a sequence $t_{1}, t_{2}, \cdots$, and then orthogonalize the sequence

$$
f_{t_{1}}-f_{0}, f_{t_{2}}-f_{0}, \cdots
$$

by the Gram-E. Schmidt procedure, thus obtaining the (finite or enumerably infinite) normalized orthogonal set

$$
\phi_{1}, \phi_{2}, \cdots \text {. }
$$

Thus

$$
\phi_{i}=\sum_{j=1}^{i} \alpha_{i j}\left(f_{i_{j}}-f_{0}\right), \quad \text { all } \alpha_{i j} \text { are real numbers. }
$$

The $f_{t}-f_{0}, t$ rational, are linear aggregates of the $\phi_{i}$ 's, hence (owing to $f_{i}$ 's continuity in $t$ ) all $f_{t}-f_{0},-\infty<t<\infty$, are limit points of such linear aggregates. Therefore all $f_{t}-f_{0}$ belong to the closed, linear set which is spanned by $\phi_{1}, \phi_{2}, \cdots$, and which therefore coincides with $\mathfrak{S}_{1}$. Hence

$$
f_{t}-f_{0}=\sum_{i} \beta_{i}(t) \phi_{i}
$$

where $\beta_{i}(t)$ are real continuous functions of $t$.

Combining (2.4) and (2.5) we get

$$
f_{t}-f_{0}=\sum_{i} \beta_{i}(t)\left\{\sum_{j=1}^{i} \alpha_{i j}\left(f_{t_{j}}-f_{0}\right)\right\},
$$

that is

$$
\left\|\left(f_{t}-f_{0}\right)-\sum_{i} \beta_{i}(t)\left\{\sum_{j=1}^{i} \alpha_{i j}\left(f_{t j}-f_{0}\right)\right\}\right\|^{2}=0 .
$$


Consider the equation

$$
f_{t+8}-f_{s}=\sum_{i} \beta_{i}(t)\left\{\sum_{j=1}^{i} \alpha_{i j}\left(f_{t_{j}+8}-f_{s}\right)\right\},
$$

and its equivalent

$$
\left\|\left(f_{t+s}-f_{s}\right)-\sum_{i} \beta_{i}(t)\left\{\sum_{j=1}^{i} \alpha_{i j}\left(f_{t_{j+s}}-f_{s}\right)\right\}\right\|^{2}=0 .
$$

$\left(2.7^{\prime}\right)$ can be written as a relation of the

$$
\left(f_{t^{\prime}+8}-f_{8}, f_{t^{\prime \prime}+8}-f_{8}\right) \quad \text { for } t^{\prime}, t^{\prime \prime}=t, t_{1}, t_{2}, \cdots,
$$

and the

$$
\beta_{i}(t), \quad \alpha_{i j}
$$

By (2.3) this means a relation of the

$$
G\left(t^{\prime}, t^{\prime \prime}\right) \quad \text { for } t^{\prime}, t^{\prime \prime}=t, t_{1}, t_{2}, \cdots,
$$

and the

$$
\beta_{i}(t), \quad \alpha_{i j} .
$$

Hence $\left(2.7^{\prime}\right)$, and consequently $\left(2.6^{\prime}\right)$, are independent of $s$. But for $s=0$ $\left(2.6^{\prime}\right),\left(2.7^{\prime}\right)$ coincide with $(2.6),(2.7)$, and hence are true. Therefore they hold for all $s$.

Let

$$
\phi_{i}(s)=\sum_{j=1}^{i} \alpha_{i j}\left(f_{t_{j+8}}-f_{8}\right) .
$$

Then $\left(2.6^{\prime}\right)$ gives

$$
f_{t+s}-f_{s}=\sum_{i} \beta_{i}(t) \phi_{i}(s)
$$

The relation

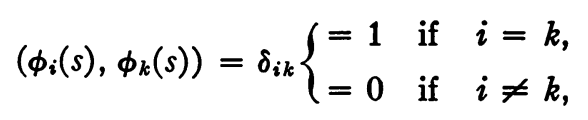

means

$$
\left(\sum_{j=1}^{i} \alpha_{i j}\left(f_{t_{j+8}}-f_{s}\right), \sum_{j=1}^{k} \alpha_{k j}\left(f_{t_{j+8}}-f_{s}\right)\right)=\delta_{i k},
$$

which is a relation of the

$$
\left(f_{t^{\prime}+8}-f_{8}, f_{t^{\prime \prime}+8}-f_{8}\right), \quad \text { for } t^{\prime}, t^{\prime \prime}=t_{1}, t_{2}, \cdots,
$$

and the 


$$
\alpha_{i j}, \quad \alpha_{k j}
$$

By (2.3) this means a relation of

$$
G\left(t^{\prime}, t^{\prime \prime}\right), \quad \text { for } t^{\prime}, t^{\prime \prime}=t_{1}, t_{2}, \cdots,
$$

and the

$$
\alpha_{i j}, \quad \alpha_{k j} .
$$

Hence (2.8) is independent of $s$. It expresses that $\phi_{1}(s), \phi_{2}(s), \cdots$ is a normalized orthogonal set. Since $\phi_{i}(0)=\phi_{i}$, this is true for $s=0$. This proves that

$$
\phi_{1}(s), \phi_{2}(s), \cdots \text { is a normalized orthogonal set for every } s \text {. }
$$

2.2. Consider a fixed $s$. The $\phi_{1}(s), \phi_{2}(s), \cdots$ span the same closed, linear set as the $f_{t_{1}+8}-f_{s}, f_{t_{2}+8}-f_{8}, \cdots$, that is, as the $f_{t+8}-f_{s}, t$ rational. Owing to the continuity of $f_{u}$ in $u$, this is the same set as spanned by the $f_{t+8}-f_{s}$, $-\infty<t<+\infty$, or, if we write $t$ for $t+s$, by the $f_{t}-f_{s},-\infty<t<+\infty$.

This set contains in particular $f_{0}-f_{s}$, hence every $f_{t}-f_{0}=\left(f_{t}-f_{s}\right)-\left(f_{0}-f_{s}\right)$. Hence it contains $\mathfrak{S}_{1}$. But $\mathfrak{S}_{1}$ contains all $f_{t}-f_{0}$ (by (2.5), along with the $\phi_{i}$ ), hence $f_{s}-f_{0}$ and the $f_{t}-f_{s}=\left(f_{t}-f_{0}\right)-\left(f_{s}-f_{0}\right)$. Hence it contains our set, too. In other words:

$$
\phi_{1}(s), \phi_{2}(s), \cdots \text { span the closed linear set } \mathfrak{S}_{1} \text { for every } s .
$$

By (2.9), (2.10) the equations

$$
U(s) \phi_{i}(0)=\phi_{i}(s),
$$$$
\text { for } i=1,2, \cdots \text {, }
$$

define a unitary transformation $U(s)$ in $\mathfrak{S}_{1}$. $\left(2.5^{\prime}\right)$ gives immediately

$$
U(s)\left(f_{t}-f_{0}\right)=f_{t+s}-f_{s},
$$

and by $\left(2.4^{\prime}\right)$ this relation (2.12) again implies (2.11).

If we write (2.12) for $t=u$ and $v$, and subtract, we get

$$
U(s)\left(f_{u}-f_{v}\right)=f_{u+s}-f_{v+s} .
$$

Since (2.12) is a special case of (2.13) $(u=t, v=0)$ we see that (2.13) is also characteristic for $U(s)$. Application of (2.13) for $s=s_{1}, s_{2}, s_{1}+s_{2}$ shows that

$$
U\left(s_{2}\right) U\left(s_{1}\right)=U\left(s_{1}+s_{2}\right) \text {. }
$$

As $f_{t_{j+s}}, f_{s}$ are continuous functions of $s$, so is $\phi_{i}(s)$ by $\left(2.4^{\prime}\right)$. Now (2.11) shows that

$$
U(s) \text { is a (strongly) continuous function of } s .
$$

2.3. Extend now $\mathfrak{S}$ to a complex unitary space $\overline{\mathfrak{S}}$, and correspondingly $\mathfrak{S}_{1}$ to $\overline{\mathfrak{S}}_{1}$. Then $U(s)$ extends to a unitary operator in $\overline{\mathfrak{S}}_{1}$. Since the extended meaning of $\|f-g\|$ and $U(s)$ remains unchanged in $\mathfrak{S}_{1}$, it is clear that (2.1), (2.12) and (2.15) remain true. 
As $\overline{\mathfrak{S}}_{1}$ is spanned by the enumerable set $f_{t_{1}}-f_{0}, f_{t_{2}}-f_{0}, \cdots$, it is separable, hence a finite-dimensional unitary or a complex Hilbert space. Now we may apply, considering (2.14), (2.15), the theorem of M. H. Stone $([6,7]$; this theorem is independent of the separability of the underlying unitary space). This theorem insures the existence of a self-adjoint operator $\bar{A}$ in $\overline{\mathfrak{S}}_{1}$, such that

$$
U(s)=\exp (i s \bar{A})
$$

Now equation (2.12) may be written as

$$
f_{t+8}=U(s)\left(f_{t}-f_{0}\right)+f_{s},
$$

or, using (2.16),

$$
f_{t+8}=\exp (i s \bar{A})\left(f_{t}-f_{0}\right)+f_{s} .
$$

2.4. Let $\bar{E}(x)$ be the resolution of unity which belongs to $\bar{A}$ in $\overline{\mathfrak{S}}_{1}$. Define the projections $\bar{F}_{n, \epsilon}, n=0, \pm 1, \pm 2, \cdots, \epsilon= \pm 1$, and $\bar{F}^{0}$ as follows:

$$
\begin{aligned}
\bar{F}_{n,+1} & =\bar{E}\left(2^{n}\right)-\bar{E}\left(2^{n-1}\right), \\
\bar{F}_{n,-1} & =\bar{E}\left(-2^{n-1}\right)-\bar{E}\left(-2^{n}\right), \\
\bar{F}^{0} & =\bar{E}(0)-\bar{E}(-0) \quad\left(E(-0)=\lim _{x<0, x \rightarrow 0} \bar{E}(x)\right) .
\end{aligned}
$$

The projection operators $\bar{F}_{n, \epsilon}, \bar{F}^{0}$ are clearly orthogonal and their sum is 1 .

Let

$\overline{\mathfrak{M}}_{n, \epsilon}$ be the closed linear set with the projection $\bar{F}_{n, \epsilon}$,

$$
(n=0, \pm 1, \pm 2, \cdots, \epsilon= \pm 1)
$$

$\overline{\mathfrak{M}}^{0}$ be the closed linear set with the projection $\bar{F}^{0}$.

Again the $\overline{\mathfrak{M}}_{n, \epsilon}, \overline{\mathfrak{M}}^{0}$ are mutually orthogonal and they span together the closed linear set $\overline{\mathfrak{S}}_{1}$. Clearly every $\overline{\mathfrak{M}}_{n, \epsilon}, \overline{\mathfrak{M}}^{0}$ reduces $\bar{A}$, and hence, by $(2.16)$, all $U(s)$, too.

Denote the projections of $f_{t}$ in $\overline{\mathfrak{M}}_{n, \epsilon}$, and $\overline{\mathfrak{M}}^{0}$, by $\bar{f}_{n, \epsilon / t}$ and $\bar{f}_{t}^{0}$, respectively.

2.5. Let us consider first the situation in $\overline{\mathfrak{M}}^{0}$. Here clearly $\bar{A}=0$, and (2.17) gives

$$
\bar{f}_{t+s}^{0}=\bar{f}_{t}^{0}-\bar{f}_{0}^{0}+\bar{f}_{s}^{0}
$$

or

$$
\left(\vec{f}_{t+s}^{0}-\vec{f}_{0}^{0}\right)=\left(\vec{f}_{t}^{0}-\vec{f}_{0}^{0}\right)+\left(\vec{f}_{s}^{0}-\vec{f}_{0}^{0}\right) .
$$

This, and the $t$-continuity of $\bar{f}_{t}$, give immediately

$$
\vec{f}_{t}^{0}-\vec{f}_{0}^{0}=t\left(\vec{f}_{1}^{0}-\vec{f}_{0}^{0}\right),
$$

that is, 


$$
\bar{f}_{t}^{0}=t \bar{\omega}^{0}+\bar{f}_{0}^{0}
$$

$\bar{\omega}^{0}$ being fixed.

2.6. Let us consider next the situation in $\overline{\mathfrak{M}}_{n, \mathrm{e}}$. Here the spectrum $S_{n, \mathrm{e}}$ of $\bar{A}$ lies clearly between $2^{n-1}$ and $2^{n}$, and between $-2^{n}$ and $-2^{n-1}$ respectively. Hence $x \in S_{n, \mathrm{e}}$ implies $2^{n-1} \leqq|x| \leqq 2^{n}$.

Assume

$$
0<|t| \leqq \pi / 2^{n} \text {. }
$$

Then $x \in S_{n, \epsilon}$ implies $0<2^{n-1}|t| \leqq|t x| \leqq \pi$. Hence the function

$$
f_{t}(x)=(\exp (i t x)-1)^{-1}
$$

is everywhere defined, continuous, and bounded in $x \in S_{n, \epsilon}$. Hence we may form $f_{t}(\bar{A})$. As $(\exp (i t x)-1) f_{t}(x)=f_{t}(x)(\exp (i t x)-1)=1$ for all $x \in S_{n, \epsilon}$, we have $(\exp (i t \bar{A})-1) f_{t}(\bar{A})=f_{t}(\bar{A})(\exp (i t \bar{A})-1)=1$. Hence $f_{t}(\bar{A})$ is the inverse of $\exp (i t \bar{A})-1$.

We have thus proved that

$$
(\exp (i t \bar{A})-1)^{-1} \text { exists and is bounded. }
$$

Consider now (2.17) for $t, s$ and for $s, t$. As the left side is the same in both cases, the right sides are equal, too, giving

$$
\exp (i s \bar{A})\left(\bar{f}_{n \epsilon / t}-\bar{f}_{n \epsilon / 0}\right)+\bar{f}_{n \epsilon / 8}=\exp (i t \bar{A})\left(\bar{f}_{n \epsilon / 8}-\bar{f}_{n \epsilon / 0}\right)+\bar{f}_{n \epsilon / t},
$$

that is

$$
\{\exp (i s \bar{A})-1\}\left(\bar{f}_{n \epsilon / t}-\bar{f}_{n \epsilon / 0}\right)=\{\exp (i t \bar{A})-1\}\left(\bar{f}_{n \epsilon / 8}-\bar{f}_{n \epsilon / 0}\right) .
$$

Assume (2.22) for both $t$ and $s$. Since $\exp (i t \bar{A})-1$ and $\exp (i s \bar{A})-1$ commute, and by (2.23) for $t$ and $s$, we may write this as

$$
\{\exp (i t \bar{A})-1\}^{-1}\left(\bar{f}_{n \epsilon / t}-\bar{f}_{n \epsilon / 0}\right)=\{\exp (i s \bar{A})-1\}^{-1}\left(\bar{f}_{n \epsilon / s}-\bar{f}_{n \epsilon / 0}\right) .
$$

That is,

$$
\{\exp (i t \bar{A})-1\}^{-1}\left(\bar{f}_{n \epsilon / t}-\bar{f}_{n \epsilon / 0}\right)=\bar{v}_{n \epsilon},
$$

where $\bar{v}_{n \in}$ is fixed. But this may be written as

$$
\bar{f}_{n \epsilon / t}=\{\exp (i t \bar{A})-1\} \bar{v}_{n \epsilon}+\bar{f}_{n \epsilon / 0}, \quad \bar{v}_{n \epsilon} \text { constant. }
$$

This holds provided $t$ satisfies (2.22). Since it is obviously true for $t=0$, it holds whenever $|t| \leqq \pi / 2^{n}$. But if (2.24) holds for both $t$ and $s$ then (2.17) extends it to $t+s$. Hence (2.24) holds for all $t$.

We may now formulate (2.21), (2.24) as follows:

The projections of $f_{t}-f_{0}$ in $\overline{\mathfrak{M}}^{0}$ and $\overline{\mathfrak{M}}_{n, \epsilon}$ are $t \bar{\omega}^{0}$ and $\{\exp (i \bar{A})-1\} \bar{v}_{n \epsilon}$ respectively, the $\bar{\omega}^{0}, \bar{v}_{n \epsilon}$ being fixed elements of $\overline{\mathfrak{M}}^{0}$ and $\overline{\mathfrak{M}}_{n, \epsilon}$ respectively. 
2.7. We know that $\overline{\mathfrak{S}}_{1}$ is the closed linear set spanned by the mutually orthogonal sets $\overline{\mathfrak{M}}^{0}$ and $\overline{\mathfrak{M}}_{n, \epsilon}(n=0, \pm 1, \pm 2, \cdots, \epsilon= \pm 1)$. As $f_{\imath}-f_{0}$ is in $\mathfrak{S}_{1}$, we see that (2.25) implies

$$
f_{t}-f_{0}=t \bar{\omega}^{0}+\sum_{n=-\infty}^{\infty} \sum_{\epsilon= \pm 1}\{\exp (i t \bar{A})-1\} \bar{v}_{n \epsilon},
$$

the convergence of the right side being certain for all $t$.

Since the addends of the right side of (2.26) are mutually orthogonal, we conclude that

$$
\left\|f_{t}-f_{0}\right\|^{2}=t^{2}\left\|\bar{\omega}^{0}\right\|^{2}+\sum_{n=-\infty}^{\infty} \sum_{\epsilon= \pm 1}\left\|\{\exp (i t \bar{A})-1\} \bar{v}_{n \epsilon}\right\|^{2} .
$$

But for each term of this sum we have

$$
\begin{aligned}
\left\|\{\exp (i t \bar{A})-1\} \bar{v}_{n \epsilon}\right\|^{2} & =\left(\{\exp (i t \bar{A})-1\} \bar{v}_{n \epsilon},\{\exp (i t \bar{A})-1\} \bar{v}_{n \epsilon}\right) \\
& =\left(\{\exp (i t \bar{A})-1\} *\{\exp (i t \bar{A})-1\} \bar{v}_{n \epsilon}, \bar{v}_{n \epsilon}\right) .
\end{aligned}
$$

By well known properties of functions of Hermitian operators, and since

$$
\begin{aligned}
\overline{(\exp (i t x)-1)}(\exp (i t x)-1) & =(\exp (-i t x)-1)(\exp (i t x)-1) \\
& =2-\exp (i t x)-\exp (-i t x)=2(1-\cos t x),
\end{aligned}
$$

the above expression is equal to

that is, to

$$
\left(2(1-\cos (\bar{t} \bar{A})) \bar{v}_{n \epsilon}, \bar{v}_{n \epsilon}\right),
$$

$$
\int 2(1-\cos t x) d\left(\left\|\bar{E}(x) \bar{v}_{n \epsilon}\right\|^{2}\right) \text {. }
$$

It suffices to extend this Stieltjes integral from $2^{n-1}$ to $2^{n}$, if $\epsilon=+1$; and from $-2^{n}$ to $-2^{n-1}$ if $\epsilon=-1$. Denote this interval by $I_{n, \mathrm{e}}$.

We have thus obtained

$$
F^{2}(t)=\left\|f_{t}-f_{0}\right\|^{2}=t^{2}\left\|\bar{\omega}^{0}\right\|^{2}+\sum_{n=-\infty}^{\infty} \sum_{\epsilon= \pm 1} \int_{I_{n, \epsilon}} 2(1-\cos t x) d\left(\left\|\bar{E}(x) \bar{v}_{n \epsilon}\right\|^{2}\right)
$$

Integrating this relation from 0 to 1 we obtain

$$
\int_{0}^{1} F^{2}(t) d t=\frac{1}{3}\left\|\bar{\omega}^{0}\right\|^{2}+\sum_{n=-\infty}^{\infty} \sum_{\epsilon= \pm 1} \int_{I_{n, \epsilon}} 2\left(1-\frac{\sin x}{x}\right) d\left(\left\|\bar{E}(x) \bar{v}_{n \epsilon}\right\|^{2}\right) .
$$

Since

$$
2\left(1-\frac{\sin x}{x}\right)\left\{\begin{array}{lll}
\geqq \frac{1}{4} x^{2}, & \text { if } & |x| \leqq 1 \\
\geqq \frac{1}{4}, & \text { if } & |x| \geqq 1,
\end{array}\right.
$$

we derive from (2.28) the finiteness of the following expression: 


$$
\sum_{n=-\infty}^{0} \sum_{\epsilon= \pm 1} \int_{I_{n, \epsilon}} \frac{1}{4} x^{2} d\left\|\bar{E}(x) \bar{v}_{n \epsilon}\right\|^{2}+\sum_{n=1}^{\infty} \sum_{\epsilon= \pm 1} \int_{I_{n, \epsilon}} \frac{1}{4} d\left\|\bar{E}(x) \bar{v}_{n \epsilon}\right\|^{2}
$$

2.8. We may now readily complete a proof of Theorem 1 by showing that the last side of (2.27) can be put in the form (1.4). Indeed, define two nondecreasing functions $\beta_{-}(x)(-\infty<x<0)$ and $\beta_{+}(x)(0<x<+\infty)$ as follows: Set

$$
\beta_{-}(x)=\left\|\bar{E}(x) \bar{v}_{0,-1}\right\|^{2} \text { in } I_{0,-1}\left(-1 \leqq x \leqq-\frac{1}{2}\right),
$$

and extend the definition successively to $I_{1,-1}, I_{2,-1}, \cdots$, and also to $I_{-1,-1}, I_{-2,-1}, \cdots$, in such a way that

(i) $\beta_{-}(x)=\left\|\bar{E}(x) \bar{v}_{n,-1}\right\|^{2}+$ const., in $I_{n,-1}\left(-2^{n} \leqq x \leqq-2^{n-1}\right)$,

(ii) the constants are successively determined by the requirement that we have agreement of values of $\beta_{-}(x)$, at every point $x=-2^{n}$, resulting from this function's definition (i) in the adjoining intervals $I_{n,-1}$ and $I_{n+1,-1}$.

Set similarly

$$
\beta_{+}(x)=\left\|\bar{E}(x) \bar{v}_{0,1}\right\|^{2} \quad \text { in } \quad I_{0,1} \quad\left(\frac{1}{2} \leqq x \leqq 1\right),
$$

and define successively

$$
\beta_{+}(x)=\left\|\bar{E}(x) \bar{v}_{n, 1}\right\|^{2}=\text { const., } \quad \text { in } I_{n, 1}\left(2^{n-1} \leqq x \leqq 2^{n}\right),
$$

determining the constants successively by the same requirement as above. The finite expression $\left(2.28^{\prime}\right)$ now becomes

$$
\int_{-1}^{-0} \frac{1}{4} x^{2} d \beta_{-}(x)+\int_{+0}^{1} \frac{1}{4} x^{2} d \beta_{+}(x)+\int_{-\infty}^{-1} \frac{1}{4} d \beta_{-}(x)+\int_{1}^{\infty} \frac{1}{4} d \beta_{+}(x),
$$

and (2.27) may be written as

$$
F^{2}(t)=t^{2}\left\|\bar{\omega}^{0}\right\|^{2}+\int_{-\infty}^{-0} 2(1-\cos t x) d \beta_{-}(x)+\int_{+0}^{\infty} 2(1-\cos t x) d \beta_{+}(x) .
$$

Setting

$$
\alpha(x)=2 \beta_{+}(x)-2 \beta_{-}(-x), \quad 0<x<\infty,
$$

this becomes

$$
F^{2}(t)=t^{2}\left\|\bar{\omega}^{0}\right\|^{2}+\int_{+0}^{\infty}(1-\cos t x) d \alpha(x)
$$

where $\alpha(x)$ is non-decreasing such that

$$
\begin{aligned}
\alpha(\infty) & =2 \beta_{+}(\infty)-2 \beta_{-}(-\infty) \\
\int_{+0}^{1} x^{2} d \alpha(x) & =2 \int_{+0}^{1} x^{2} d \beta_{+}(x)+2 \int_{-1}^{-0} x^{2} d \beta_{-}(x)
\end{aligned}
$$


are finite, on account of the finiteness of $\left(2.28^{\prime \prime}\right)$. If we introduce a new monotone function by setting

we have

$$
\gamma(0)=0, \quad \gamma(x)=\int_{+0}^{x} 2 x^{2} d \alpha(2 x), \quad x>0,
$$

$$
\int_{+0}^{\infty}(1-\cos t x) d \alpha(x)=\int_{+0}^{\infty} \frac{1-\cos 2 t x}{2 x^{2}} 2 x^{2} d \alpha(2 x)=\int_{+0}^{\infty} \frac{\sin ^{2} t x}{x^{2}} d \gamma(x) .
$$

By changing, finally, the value of $\gamma(0)$ from 0 to $-\left\|\bar{\omega}^{0}\right\|^{2}$, we see that $\left(2.27^{\prime}\right)$ now assumes the form (1.4). Also (1.5) is satisfied, since $\alpha(x)$ is bounded.

Part III. Second proof and extension of Fundamental theorem BY THE THEORY OF POSITIVE DEFINITE FUNCTIONS

3.1. We know that the class of screw functions of Hilbert space is identical with the class of continuous, even, non-negative functions $F(t)(F(0)=0)$ such that the metric transform $F\left(E_{1}\right)$ is isometrically imbeddable in $\mathfrak{S}$ (Introduction, §1). Let us denote this class of functions by the symbol $\Pi\left(E_{1}\right)$. We now extend the problem of determining the class $\Pi\left(E_{1}\right)$, as follows: Determine the class $\Pi\left(E_{m}\right)$ of functions $F(t)$ such that the metric transform $F\left(E_{m}\right)$ be isometrically imbeddable in $\mathfrak{S}\left(1 \leqq m \leqq \infty, E_{\infty}=\mathfrak{S}\right)$. (See [5, Introduction and \$5.2].)

The definition of $\Pi\left(E_{m}\right)$ implies the relations

$$
\Pi\left(E_{1}\right) \supset \Pi\left(E_{2}\right) \supset \cdots \supset \Pi\left(E_{m}\right) \supset \cdots \supset \Pi(\mathfrak{S}),
$$

showing that we have to deal with an infinite sequence of non-increasing subsets of $\Pi\left(E_{1}\right)$. The class $\Pi(\mathfrak{S})$ was found to be identical with the class of functions $F(t)$ whose squares are of the form

$$
F^{2}(t)=\int_{0}^{\infty} \frac{1-e^{-t^{2} u}}{u} d \gamma(u),
$$

where $\gamma(u)$ is non-decreasing for $u \geqq 0$ and such that $\int_{1}^{\infty} u^{-1} d \gamma(u)$ exists [5, $\S 5.4$, Theorem 6]. We shall now determine the classes $\Pi\left(E_{m}\right),(1 \leqq m<\infty)$.

The statement of our result requires the integral function

$$
\begin{aligned}
\Omega_{m}(t) & =1-\frac{t^{2}}{2 \cdot m}+\frac{t^{4}}{2 \cdot 4 \cdot m(m+2)}-\frac{t^{6}}{2 \cdot 4 \cdot 6 \cdot m(m+2)(m+4)}+\cdots \\
& =\Gamma\left(\frac{m}{2}\right)\left(\frac{2}{t}\right)^{(m-2) / 2} J_{(m-2) / 2}(t) .
\end{aligned}
$$

In particular

$$
\Omega_{1}(t)=\cos t, \quad \Omega_{2}(t)=J_{0}(t), \quad \Omega_{3}(t)=\sin t / t .
$$


THEOREM 7. The class $\Pi\left(E_{m}\right)$ of non-negative continuous functions $F(t)$ $(t \geqq 0, F(0)=0)$ such that the metric transform $F\left(E_{m}\right)$ be isometrically imbeddable in $\mathfrak{S}$, is identical with the class of functions whose squares are of the form

$$
F^{2}(t)=\int_{0}^{\infty} \frac{1-\Omega_{m}(t u)}{u^{2}} d \gamma(u)
$$

where $\gamma(u)$ is non-decreasing for $u \geqq 0, \gamma(0)=0$, and such that

$$
\int_{1}^{\infty} \frac{d \gamma(u)}{u^{2}} \text { exists. }
$$

If $m=1$ we obtain our Theorem 1 on screw functions, since $1-\Omega_{1}(t u)$ $=1-\cos t u=2 \sin ^{2}(t u / 2)$.

The reasoning which leads to the characterization of the class $\Pi\left(E_{1}\right)$ given by Lemma $1, \S 1.1$, extends, of course, to the class $\Pi\left(E_{m}\right)$ and gives the following statement:

$A$ continuous non-negative function $F(t)(t \geqq 0, F(0)=0)$ belongs to $\Pi\left(E_{m}\right)$ if and only if it satisfies the inequality

$$
\sum_{i, k=1}^{n}\left\{F^{2}\left(P_{0} P_{i}\right)+F^{2}\left(P_{0} P_{k}\right)-F^{2}\left(P_{i} P_{k}\right)\right\} \rho_{i} \rho_{k} \geqq 0,
$$

for any points $P_{0}, \cdots, P_{n}$ of $E_{m}$ and arbitrary real $\rho_{i}(n=2,3, \cdots)$, the quantities $P_{i} P_{k}$ denoting euclidean distances in $E_{m}$.

We refer to $[5, \S 8.1]$, for a simple proof, based on the statement above, that any $F(t)$ given by (3.2) belongs to $\Pi\left(E_{m}\right)$.

3.2. The proof of the converse, i.e., that formula (3.2) furnishes all elements of $\Pi\left(E_{m}\right)$, requires the following limit theorem:

LEMMA 2. Let

$$
f_{n}(t)=\int_{0}^{\infty} \frac{1-\Omega_{m}(t u)}{u^{2}} d \gamma_{n}(u), \quad n=1,2,3, \cdots,
$$

be a sequence of functions with non-decreasing $\gamma_{n}(u),\left(\gamma_{n}(0)=0\right)$, such that all integrals $\int_{1}^{\infty} u^{-2} d \gamma_{n}(u)$ exist. Let $f_{n}(t)$ converge, as $n \rightarrow \infty$, uniformly in any finite interval, to a function $f(t)$. We indicate this type of convergence by the relation

$$
f_{n}(t) \rightarrow f(t) \text {. }
$$

Then $f(t)$ is also of the form

$$
f(t)=\int_{0}^{\infty} \frac{1-\Omega_{m}(t u)}{u^{2}} d \gamma(u), \quad \gamma(u) \uparrow, \gamma(0)=0, \int_{1}^{\infty} u^{-2} d \gamma(u) \text { exists, }
$$

and 


$$
\gamma_{n}(u) \rightarrow \gamma(u)\left({ }^{2}\right)
$$

$$
\int_{a}^{\infty} \frac{d \gamma_{n}(u)}{u^{2}} \rightarrow \int_{a}^{\infty} \frac{d \gamma(u)}{u^{2}} \text { in all continuity points } u=a>0 \text { of } \gamma(u)
$$

Conversely, if the relations (3.8) and (3.9) hold and $f_{n}(t), f(t)$ are defined by (3.5) and (3.7), then (3.6) holds.

Postponing a proof of this limit theorem we shall now use it to establish Theorem 7.

Let $F(t) \in \Pi\left(E_{m}\right)$, that is, $F\left(E_{m}\right)$ be imbeddable in $\mathfrak{S}$. By $[5, \S 5.3$, Corollary 1] we conclude that the functions exp $\left\{-\lambda F^{2}(t)\right\},(\lambda>0)$, belong to the class $\mathfrak{P}\left(E_{m}\right)$ of functions which are positive definite in $E_{m}$. By $[5, \S 1.2$, Theorem 1], we are allowed to set

$$
\exp \left\{-\lambda F^{2}(t)\right\}=\int_{0}^{\infty} \Omega_{m}(t u) d \alpha(u, \lambda), \quad \lambda>0,
$$

$\alpha(u, \lambda)$ being a family of non-decreasing functions, $\alpha(0, \lambda)=0, \alpha(\infty, \lambda)=1$. Now

$$
\frac{1-\exp \left\{-\lambda F^{2}(t)\right\}}{\lambda}=\frac{1}{\lambda} \int_{0}^{\infty}\left[1-\Omega_{m}(t u)\right] d \alpha(u, \lambda),
$$

and if we set

we obtain

$$
\beta(u, \lambda)=\frac{1}{\lambda} \int_{0}^{u} u^{2} d \alpha(u, \lambda),
$$

$$
f(t, \lambda)=\frac{1-\exp \left\{-\lambda F^{2}(t)\right\}}{\lambda}=\int_{0}^{\infty} \frac{1-\Omega_{m}(t u)}{u^{2}} d \beta(u, \lambda) .
$$

On the other hand,

$$
\lim _{\lambda \rightarrow+0} f(t, \lambda)=F^{2}(t)
$$

holds uniformly in any finite interval. For, if $0 \leqq t \leqq T$, we have $F^{2}(t)<K$ (since $F(t)$ is continuous) and therefore

$$
\begin{aligned}
\left|\exp \left\{-\lambda F^{2}(t)\right\}-1+\lambda F^{2}(t)\right| & =\left|\lambda^{2} F^{4}(t) / 2 !-\lambda^{3} F^{6}(t) / 3 !+\cdots\right| \\
& \leqq \lambda^{2} K^{2} e^{\lambda K}
\end{aligned}
$$

or

(2) Here and throughout this paper a limiting relation $\gamma_{n}(u) \rightarrow \gamma(u)$ involving monotone functions is assumed to hold for all values of $u$ which are continuity points of the limiting function $\gamma(u)$. 


$$
\left|\frac{1-\exp \left\{-\lambda F^{2}(t)\right\}}{\lambda}-F^{2}(t)\right| \leqq \lambda K^{2} e^{\lambda K},
$$

a bound which is independent of $t$ and tends to zero with $\lambda$. Setting $f_{n}(t)$ $=f(t, 1 / n)$ we therefore have $f_{n}(t) \rightarrow F^{2}(t)$ and Lemma 2 is applicable, on account of (3.10), showing that $F^{2}(t)$ is of the form (3.2).

3.3. In this proof of Theorem 7 only a part of Lemma 2 was used, namely that (3.5) and (3.6) imply (3.7). We have stated and shall prove the more inclusive limit theorem for two reasons. First, our limit theorem implies that $\gamma(u)$ of (3.2) is uniquely defined by $F(t)$. Second, a proof of the complete statement is hardly more complicated than a proof of the partial statement actually used above.

Lemma 2 will be a direct consequence of the following analogous limit theorem involving a simpler kernel under the integral sign.

LEMMA 3. Let

$$
\phi_{n}(t)=\int_{0}^{\infty} \frac{1-e^{-u t}}{u} d \beta_{n}(u), \quad n=1,2,3, \cdots,
$$

be a sequence of functions with non-decreasing $\beta_{n}(u)\left(\beta_{n}(0)=0\right)$, such that all integrals $\int_{1}^{\infty} u^{-1} d \beta_{n}(u)$ exist. Let $\phi_{n}(t)$ converge, as $n \rightarrow \infty$, to a function $\phi(t)$, uniformly in any finite interval $0 \leqq t \leqq t_{0}$, i.e.,

$$
\phi_{n}(t) \rightarrow \phi(t) \text {. }
$$

Then $\phi(t)$ is also of the form

$$
\phi(t)=\int_{0}^{\infty} \frac{1-e^{-u t}}{u} d \beta(u), \quad \beta(u) \uparrow, \beta(0)=0, \int_{1}^{\infty} u^{-1} d \beta(u) \text { exists }
$$

and

$$
\begin{aligned}
\beta_{n}(u) & \rightarrow \beta(u) \\
\int_{a}^{\infty} \frac{d \beta_{n}(u)}{u} & \rightarrow \int_{a}^{\infty} \frac{d \beta(u)}{u} \text { in all continuity points } u=a>0 \text { of } \beta(u) .
\end{aligned}
$$

Conversely, if the relations (3.14) and (3.15) hold and $\phi_{n}(t), \phi(t)$ are defined by (3.11) and (3.13), then (3.12) holds $\left.{ }^{3}\right)$.

The $\phi_{n}(t)$ belong to the class $T$ defined in $[5, \S 4]$. It was shown there (Lemma 5) that (3.12) implies $\phi(t) \in T$, hence (3.13). It was furthermore

(3) That the condition (3.15) cannot be dispensed with is shown by the example of $\beta_{n}(u)=0$ if $0 \leqq u<n, \beta_{n}(u)=n$ if $u \geqq n, \beta(u) \equiv 0$, hence $\phi_{n}(t)=1-e^{-n t}, \phi(t) \equiv 0$. Now (3.14) holds but not (3.12). Note that the condition (3.15) is not satisfied. 
shown by means of the Vitali-Porter convergence theorem that $\phi_{n}(t)$ and

$$
\phi_{n}^{\prime}(t)=\int_{0}^{\infty} e^{-u t} d \beta_{n}(u)
$$

converge to $\phi(t)$ and $\phi^{\prime}(t)$, respectively, uniformly in any finite closed domain inside the half-plane $\Re t>0$ of the complex variable $t=\sigma+i \tau$. In particular we therefore have

$$
\phi_{n}^{\prime}(1+i \tau)=\int_{0}^{\infty} e^{-u} e^{-i u \tau} d \beta_{n}(u) \rightarrow \phi^{\prime}(1+i \tau)=\int_{0}^{\infty} e^{-u} e^{-i u \tau} d \beta(u),
$$

uniformly in any finite $\tau$-interval. In terms of the new monotone and bounded functions

$$
\alpha_{n}(u)=\int_{0}^{u} e^{-u} d \beta_{n}(u), \quad \alpha(u)=\int_{0}^{u} e^{-u} d \beta(u),
$$

the relation (3.16) becomes

$$
\int_{0}^{\infty} e^{-i u \tau} d \alpha_{n}(u) \rightarrow \int_{0}^{\infty} e^{-i u \tau} d \alpha(u)
$$

Now we may apply a limit theorem of Paul Lévy and conclude that

$$
\alpha_{n}(u) \rightarrow \alpha(u)
$$

$[1$, p. 197]. But (3.17) may be inverted:

$$
\beta_{n}(u)=\int_{0}^{u} e^{u} d \alpha_{n}(u), \quad \beta(u)=\int_{0}^{u} e^{u} d \alpha(u) .
$$

Now (3.18) implies the desired relation (3.14).

There remains the proving of (3.15). On one hand (3.14) implies

$$
\int_{0}^{a} \frac{1-e^{-t u}}{u} d \beta_{n}(u) \rightarrow \int_{0}^{a} \frac{1-e^{-t u}}{u} d \beta(u), \quad t \geqq 0,
$$

and therefore (3.11), (3.13) and (3.12) imply

$$
\int_{a}^{\infty} \frac{1-e^{-t u}}{u} d \beta_{n}(u) \rightarrow \int_{a}^{\infty} \frac{1-e^{-t u}}{u} d \beta(u), \quad t \geqq 0 .
$$

On the other hand, the obvious inequalities

$$
\left(1-e^{-t a}\right) \int_{a}^{\infty} \frac{d \beta_{n}(u)}{u} \leqq \int_{a}^{\infty} \frac{1-e^{-t u}}{u} d \beta_{n}(u) \leqq \int_{a}^{\infty} \frac{d \beta_{n}(u)}{u}, t \geqq 0,
$$

imply 


$$
\int_{a}^{\infty} \frac{1-e^{-t u}}{u} d \beta_{n}(u) \leqq \int_{a}^{\infty} \frac{d \beta_{n}(u)}{u} \leqq \frac{1}{1-e^{-t a}} \int_{a}^{\infty} \frac{1-e^{-t u}}{u} d \beta_{n}(u), t>0 .
$$

As $n \rightarrow \infty$ we get by (3.19)

As $t \rightarrow \infty$ this gives

$$
\begin{aligned}
\int_{a}^{\infty} \frac{1-e^{-t u}}{u} d \beta(u) & \leqq \lim \inf \int_{a}^{\infty} \frac{d \beta_{n}(u)}{u} \leqq \lim \sup \int_{a}^{\infty} \frac{d \beta_{n}(u)}{u} \\
& \leqq \frac{1}{1-e^{-t a}} \cdot \int_{a}^{\infty} \frac{1-e^{-t u}}{u} d \beta(u) .
\end{aligned}
$$

$$
\int_{a}^{\infty} \frac{d \beta(u)}{u} \leqq \lim \inf \int_{a}^{\infty} \frac{d \beta_{n}(u)}{u} \leqq \lim \sup \int_{a}^{\infty} \frac{d \beta_{n}(u)}{u} \leqq \int_{a}^{\infty} \frac{d \beta(u)}{u},
$$

which proves (3.15).

In order to prove the converse of Lemma 3, we have to show that (3.11), (3.13), (3.14), and (3.15) imply (3.12). For a given $\epsilon$, we can choose a positive $a$, continuity point of $\beta(u)$, such that

$$
\int_{a}^{\infty} u^{-1} d \beta_{n}(u)<\epsilon, \quad \int_{a}^{\infty} u^{-1} d \beta(u)<\epsilon,
$$

provided $n$ is sufficiently large. But then

$$
\int_{0}^{a} \frac{1-e^{-t u}}{u} d \beta_{n}(u), \quad \int_{0}^{a} \frac{1-e^{-t u}}{u} d \beta(u),
$$

differ from $\phi_{n}(t)$ and $\phi(t)$ respectively by less than $\epsilon$. Hence

$$
\left|\phi_{n}(t)-\phi(t)\right|<2 \epsilon+\left|\int_{0}^{a} \frac{1-e^{-t u}}{u} d \beta_{n}(u)-\int_{0}^{a} \frac{1-e^{-t u}}{u} d \beta(u)\right|,
$$

provided $n$ is sufficiently large. On the other hand, (3.14) implies

$$
\int_{0}^{a} \frac{1-e^{-t u}}{u} d \beta_{n}(u) \rightarrow \int_{0}^{a} \frac{1-e^{-t u}}{u} d \beta(u)
$$

that is,

$$
\left|\int_{0}^{a} \frac{1-e^{-t u}}{u} d \beta_{n}(u)-\int_{0}^{a} \frac{1-e^{-t u}}{u} d \beta(u)\right|<\epsilon,
$$

in an arbitrarily given interval $0 \leqq t \leqq t_{0}$, provided $n$ is large enough. Hence $\left|\phi_{n}(t)-\phi(t)\right|<3 \epsilon$, in the interval $0 \leqq t \leqq t_{0}$, provided $n$ is large enough. This is precisely identical to (3.12).

By a similar argument we can prove the converse part of Lemma 2. We 
may, therefore, consider the converse part of Lemma 2 as already established and shall use it in proving the direct part of that lemma.

3.4. In order to prepare a proof of Lemma 2 we need the following statement:

The assumptions (3.5) and (3.6), of Lemma 2, imply the existence of two constants $K$ and $H$, independent of $n$ and $t$, such that $f_{n}(t)<K t^{2}+H, t \geqq 0, n=1,2,3, \cdots$.

Assume first that $m \geqq 2$. Since

$$
-1<\Omega_{m}(t)<1, \quad(t>0) ; \quad \lim _{t \rightarrow \infty} \Omega_{m}(t)=0,
$$

we see that (3.5) and (3.6) imply (for $t=1$ ) that the two expressions

$$
\int_{0}^{1} \frac{1-\Omega_{m}(u)}{u^{2}} d \gamma_{n}(u), \quad \int_{1}^{\infty}\left[1-\Omega_{m}(u)\right] \frac{d \gamma_{n}(u)}{u^{2}}
$$

are both bounded, as $n \rightarrow \infty$. Since the integrands $\left[1-\Omega_{m}(u)\right] / u^{2}$, and $1-\Omega_{m}(u)$, have positive lower bounds in their respective intervals of integration $(0,1)$ and $(1, \infty)$, we see that

$$
\int_{0}^{1} d \gamma_{n}(u)=\gamma_{n}(1), \quad \int_{1}^{\infty} \frac{d \gamma_{n}(u)}{u^{2}}
$$

also are bounded, as $n \rightarrow \infty$. On the other hand, it is clear from the power series expansion (3.1), that there is a constant $c$, such that

$$
1-\Omega_{m}(t) \leqq c t^{2},
$$

provided $t \geqq 0$. Hence, by (3.21),

$$
\begin{aligned}
f_{n}(t) & =\int_{0}^{1} \frac{1-\Omega_{m}(t u)}{u^{2}} d \gamma_{n}(u)+\int_{1}^{\infty} \frac{1-\Omega_{m}(t u)}{u^{2}} d \gamma_{n}(u) \\
& \leqq \int_{0}^{1} \frac{c t^{2} u^{2}}{u^{2}} d \gamma_{n}(u)+\int_{1}^{\infty} \frac{2}{u^{2}} d \gamma_{n}(u) \\
& =t^{2} c \gamma_{n}(1)+2 \int_{1}^{\infty} u^{-2} d \gamma_{n}(u)<K t^{2}+H .
\end{aligned}
$$

If $m=1$, hence $\Omega_{m}(t u)=\cos t u$, we first integrate both sides of (3.6), between 0 and 1 , obtaining the relation

$$
\int_{0}^{1} f_{n}(t) d t=\int_{0}^{\infty} \frac{1-u^{-1} \sin u}{u^{2}} d \gamma_{n}(u)=\int_{0}^{\infty} \frac{1-\Omega_{3}(u)}{u^{2}} d \gamma_{n}(u) \rightarrow \int_{0}^{1} f(t) d t .
$$

From this we derive as above that (3.21) are also bounded in the present case. The argument is then concluded as above. Thus (3.20) is established. 
3.5. We may now readily prove Lemma 2. From its assumptions (3.5), (3.6), combined with (3.20), we get

$$
f_{n}(t) \rightarrow f(t), \quad 0 \leqq f_{n}(t)<K t^{2}+H, \quad 0 \leqq f(t)<K t^{2}+H, t \geqq 0 .
$$

This implies, as readily seen, that

$$
\int_{0}^{\infty} e^{-t^{2} / 4} f_{n}(t \mu) t^{m-1} d t \rightarrow \int_{0}^{\infty} e^{-t^{2} / 4} f(t \mu) t^{m-1} d t,
$$

uniformly in $\mu$ in any finite interval $0 \leqq \mu \leqq M$. Hence also

$$
\int_{0}^{\infty} e^{-t^{2} / 4} f_{n}\left(t \lambda^{1 / 2}\right) t^{m-1} d t \rightarrow \int_{0}^{\infty} e^{-t^{2} / 4} f\left(t \lambda^{1 / 2}\right) t^{m-1} d t
$$

uniformly in any finite interval $0 \leqq \lambda \leqq L$. Taking $t \lambda^{-1 / 2}$, instead of $t$, as a new variable of integration, we see that

$$
\lambda^{-m / 2} \int_{0}^{\infty} e^{-t^{2} / 4 \lambda} f_{n}(t) t^{m-1} d t \rightarrow \lambda^{-m / 2} \int_{0}^{\infty} e^{-t^{2} / 4 \lambda} f(t) t^{m-1} d t,
$$

uniformly in $\lambda$ in any finite interval $0<\lambda \leqq L$. We shall now make use of the relation $[5$, p. 823$]$

$$
e^{-\lambda u^{2}}=c_{m} \lambda^{-m / 2} \int_{0}^{\infty} \Omega_{m}(t u) e^{-t^{2} / 4 \lambda} t^{m-1} d t, \quad \lambda>0, c_{m}=2^{1-m} \cdot[\Gamma(m / 2)]^{-1},
$$

and its particular case $(u=0)$

$$
1=c_{m} \lambda^{-m / 2} \int_{0}^{\infty} e^{-t^{2} / 4 \lambda} t^{m-1} d t
$$

If we multiply the relation

$$
f_{n}(t)=\int_{0}^{\infty} \frac{1-\Omega_{m}(t u)}{u^{2}} d \gamma_{n}(u)
$$

by $c_{m} \lambda^{-m / 2} e^{-t^{2} / 4 \lambda} t^{m-1} d t$, we get by integration

$$
\begin{aligned}
\phi_{n}(\lambda) & =c_{m} \lambda^{-m / 2} \int_{0}^{\infty} e^{-t^{2} / 4 \lambda} f_{n}(t) t^{m-1} d t=\int_{0}^{\infty} \frac{1-e^{-\lambda u^{2}}}{u^{2}} d \gamma_{n}(u) \\
& =\int_{0}^{\infty} \frac{1-e^{-\lambda u}}{u} d \gamma_{n}\left(u^{1 / 2}\right) .
\end{aligned}
$$

By (3.23) $\phi_{n}(\lambda)$ converges, as $n \rightarrow \infty$, uniformly in any interval $0<\lambda \leqq L$. As the last expression is defined and continuous for $\lambda=0$, we see that the uniform convergence extends to any closed interval $0 \leqq \lambda \leqq L$. But then, by Lemma 3 , 
we conclude that there is a monotone function $\gamma\left(u^{1 / 2}\right),(u \geqq 0)$, such that

$$
\gamma_{n}\left(u^{1 / 2}\right) \rightarrow \gamma\left(u^{1 / 2}\right), \quad \int_{a^{2}}^{\infty} \frac{d \gamma_{n}\left(u^{1 / 2}\right)}{u} \rightarrow \int_{a^{2}}^{\infty} \frac{d \gamma\left(u^{1 / 2}\right)}{u},
$$

that is,

$$
\gamma_{n}(u) \rightarrow \gamma(u), \quad \int_{a^{2}}^{\infty} \frac{d \gamma_{n}(u)}{u^{2}} \rightarrow \int_{a^{2}}^{\infty} \frac{d \gamma(u)}{u^{2}} .
$$

By the converse of Lemma 2, we may now conclude that

$$
f_{n}(t)=\int_{0}^{\infty} \frac{1-\Omega_{m}(t u)}{u^{2}} d \gamma_{n}(u) \rightarrow \int_{0}^{\infty} \frac{1-\Omega_{m}(t u)}{u^{2}} d \gamma(u) .
$$

On comparing this relation with the assumption (3.6), we derive the validity of the desired conclusion (3.7).

\section{BIBLIOGRAPHY}

1. P. Lévy, Calcul des Probabilités, Paris, 1925.

2. K. Menger, Die Metrik des Hilbertschen Raumes, Anzeiger der Akademie der Wissenschaften in Wien, Mathematisch-Naturwissenschaftliche Klasse, vol. 65 (1928), pp. 159-160.

3. I. J. Schoenberg, Remarks to Maurice Fréchet's article * . , Annals of Mathematics, (2), vol. 36 (1935), pp. 724-732.

4. - Metric spaces and positive definite functions, these Transactions, vol. 44 (1938), pp. 522-536.

5. - Metric spaces and completely monotone functions, Annals of Mathematics, (2), vol. 39 (1938), pp. 811-841.

6. M. H. Stone, Linear transformations in Hilbert space III, Proceedings of the National Academy of Sciences, vol. 16 (1930), pp. 172-175.

7. - On one-parameter unitary groups in Hilbert space, Annals of Mathematics, (2), vol. 33 (1932), pp. 643-648.

8. W. A. Wilson, On certain types of continuous transformations of metric spaces, American Journal of Mathematics, vol. 57 (1935), pp. 62-68.

INSTITUTE FOR AdVANCED STUdy, Princeton, N. J.,

Colby College,

Waterville, Me. 